\title{
Plasmon-Waveguide Resonances with Enhanced Figure of Merit and Their Potential for Anisotropic Biosensing in the Near Infrared Region
}

\author{
Said Mahajna, Michal Neumann, Ofer Eyal, and Atef Shalabney \\ Physics and Optical Engineering Department, Ort Braude College, 21982 Karmiel, Israel \\ Correspondence should be addressed to Atef Shalabney; shalabney@braude.ac.il
}

Received 22 September 2016; Accepted 15 November 2016

Academic Editor: Marco Consales

Copyright (c) 2016 Said Mahajna et al. This is an open access article distributed under the Creative Commons Attribution License, which permits unrestricted use, distribution, and reproduction in any medium, provided the original work is properly cited.

\begin{abstract}
The TM and TE guided modes in the coupled plasmon-waveguide resonance configuration are investigated in the spectral domain. Here we use the modes dispersion to study their capability for sensing in the near infrared region. It is shown that the spectral widths of the guided modes are, at least, one order of magnitude smaller than the conventional surface plasmon resonance counterpart. The enhanced sensitivity and figure of merit of the guided modes reveal their potential for sensing in the spectral interrogation method where the traditional configurations are inherently limited. Moreover, the high resolution associated with the narrow resonances and the polarization dependence make these modes very promising for anisotropic biosensing in the spectral interrogation approach. The extremely high figure of merit, large penetration depth, and propagation distance in the near infrared region open the possibility of combining the plasmon-waveguide configuration with absorption spectroscopy techniques for molecular recognition.
\end{abstract}

\section{Introduction}

A great deal of interest has been recently shown in optical sensors based on surface plasmon resonances (SPRs). These are obtained when surface plasmon waves (SPWs) are resonantly excited on the interface between thin films, made of noble metals such as silver and gold, and a dielectric medium. The SPR modes are highly sensitive to the optical properties of the adjacent dielectric medium, making them a powerful tool for biosensing and molecular detection [1-14]. The Kretschmann-Raether (KR) configuration is widely used for SPR sensing in which a prism coupler with a high refractive index (RI) is used to achieve the momentum matching condition. The main features that are often considered in SPR-based sensors are the sensitivity $\left(S_{\theta}\right.$ or $S_{\lambda}$ for angular interrogation or spectral interrogation, resp.), figure of merit (FOM), penetration depth inside the sensed medium, and the propagation distance. Although SPR sensors exhibit the highest sensitivity among those based on the evanescentwaves phenomenon, tremendous efforts have been invested in the last two decades to improve their sensitivity, FOM, and detection limit [3].

As an extension of the SPR technique, Tollin and coworkers have reported on the development of a modified design in which a dielectric layer is added on top of the thin metallic film [15-18]. In this configuration, the so-called coupled plasmon-waveguide resonance (CPWR), the dielectric layer gives rise to the appearance of guided modes in addition to the SPR excitations. Unlike the conventional SPR structure, the additional guided modes can be excited in both TE and TM polarization state. Being excited inside the dielectric top layer, which acts as the waveguide, the guided modes have large propagation distances on the analyte interface making them highly efficient for sensing. This large propagation distance is realized through the extremely narrow angular bandwidths of the resonances, which in turn increase the resolution of the measurement. Moreover, the dielectric slab mechanically and chemically protects the metallic film and increases the sensor lifetime. All these advantages made CPWR-based sensors well suited and promising for the study 
of biological species [16-18]. In a recent work, Harté et al. used the CPWR configuration to study the formation of lipid membranes and their interaction with nontoxic and toxic amyloids [19].

So far, the CPWR was mainly investigated in the angular interrogation approach in which it was proposed for sensing in the visible region [20]. The dispersion properties of the guided modes in the spectral interrogation method have not been studied and the possibility of using these resonances for sensing has been hardly explored. Furthermore, the coupling between the guided modes inside the dielectric slab and the SPWs at the metal interface are not completely understood.

In this work, we theoretically investigate the properties of the optical guided modes of the CPWR in the KR configuration. The main features and the origins of the modes that are observed in both TM and TE polarization states are studied by analyzing the dispersion landscape. We show that the guided modes have extremely narrow bandwidths with respect to the conventional SPR sensors, in particular in the near infrared (NIR) region. The large FOM, enhanced sensitivity, and the polarization dependence make these modes very promising for anisotropic sensing which is highly relevant for cells and proteins studies [19, 21-23]. Moreover, the unusual capabilities of the guided modes in the NIR region pave the way of combining SPR-based sensors with absorption spectroscopy techniques for molecular detection.

\section{Results and Discussion}

The structure under study is composed of a prism coupler made of SF11 glass which is coated with a thin silver film (Ag) and then covered with a thick $\mathrm{SiO}_{2}$ layer (see Figure 1(a)). The thicknesses of the $\mathrm{Ag}$ film and the top $\mathrm{SiO}_{2}$ layer are $d_{\mathrm{Ag}}=$ $45 \mathrm{~nm}$ and $d_{\mathrm{SiO}_{2}}=1200 \mathrm{~nm}$, respectively, as schematically shown in Figure 1(a). The thickness of the $\mathrm{Ag}$ film is optimized to maximize the efficiency of the SPR excitation at the $\mathrm{Ag} / \mathrm{SiO}_{2}$ interface. The $\mathrm{SiO}_{2}$ layer thickness, on the other hand, determines the highest order of the allowed guided modes in the interrogated spectral range. The analyte to be sensed is considered as a semi-infinite medium on the $\mathrm{SiO}_{2}$ side and has a background RI $n_{a}=1.33$ (the dispersion of the $\mathrm{SF} 11, \mathrm{Ag}$, and $\mathrm{SiO}_{2}$ is given in the supplementary materials available online at http://dx.doi.org/10.1155/2016/1898315). The optical modes appear as dips in the reflectivity versus the incident angle when the excitation wavelength is fixed.

In the spectral interrogation, on the other hand, the reflectivity is acquired as a function of the wavelength when the incident angle of the broadband source is fixed.

Figures 1(b) and 1(c) show the reflectivity in the angular mode for excitation wavelength $\lambda=632.8 \mathrm{~nm}$. The SPR mode is excited at higher angles than the guided modes due to its larger momentum. Furthermore, the plasmonic mode shows broader angular dips as it partially propagates inside the metal where it experiences large dissipation. The guided modes, on the other hand, are narrower because they are confined inside the $\mathrm{SiO}_{2}$ core and hence have larger propagation distances. Here we are able to observe simultaneously the SPR modes as well as the guided modes due to the high RI of the prism for which the SPR is obtained at reasonably low angles.

In order to have extensive insights on the modes features, we plot the dispersion of the different modes for both polarization states. The lowest branch in Figure 2(a) corresponds to the SPR, which almost exhibits the normal behavior that is pertinent to this type of excitation. The dispersion is extremely steep in the NIR region and nearly approaches the light line in free space where the SPW is less confined to the surface. In the visible region, on the other hand, the dispersion nearly saturates which implies that the waves become highly bounded to the surface and the propagation distance decreases. The upper branches in Figure 2(a) represent the dispersion of the different orders of the guided modes showing their small widths with respect to the SPR. The onset of the guided modes appears at angles larger than the critical angle, which implies that they are evanescently excited through the metal cladding. The dispersion of the TE modes is shown in Figure 2(b) and has the main features as the guided modes in the TM case, except the absence of the SPR mode which is forbidden in the TE polarization. However, their contrast is smaller than the TM case, which perhaps stems from the different influence of the metal film on the excitation of TM and TE states.

The distribution of the parallel components of the electric fields intensity inside the structure is shown in Figure 3. While the SPR mode is mainly bounded to the $\mathrm{Ag} / \mathrm{SiO}$ interface, the guided modes are concentrated inside the waveguide with a significant tail inside the analyte. We further confirmed that the spectral sensitivity of the guided modes perfectly correlates with the overlap between their modal structures and the analyte region. The spectral sensitivity $S_{\lambda}$ is defined as the shift in the resonance wavelength $\Delta \lambda_{\text {res }}$ for a finite variation in the analyte RI $\Delta n_{a}\left(S_{\lambda} \equiv \Delta \lambda_{\text {res }} / \Delta n_{a}\right)$. These findings perfectly agree with previous reports on SPR modes [24].

However, the spectral sensitivity of the SP at wavelengths smaller than $1650 \mathrm{~nm}$ is extremely low as the $\mathrm{SiO}_{2}$ waveguide thickness is much larger than the penetration depth of the SPW (see the SP field distribution in Figure 3(a)). The fields in Figure 3 were calculated at excitation wavelength $\lambda=785 \mathrm{~nm}$.

In order to assess the potential of the guided modes for sensing in the spectral interrogation approach, we compare the reflectivity spectrum of the CPWR with the conventional SPR (without the dielectric layer) for TM and TE polarization states in Figures 4(a) and 4(c). Here we tune the incident angle to obtain the resonances in the NIR region. We focus on the spectral interrogation method in this region because this approach is becoming more and more attractive due to the availability of miniature and cheap spectrometers and due to the importance in combining the SPR sensor with absorption spectroscopy for molecular recognition. In real applications, biological species are normally prepared in an aqueous buffer; therefore the analyte RI is kept as $n_{a}=1.33$.

Two sharp and intense guided modes are obtained in the TM polarization, which correspond to the $\mathrm{TM}_{0}$ and $\mathrm{TM}_{1}$ 


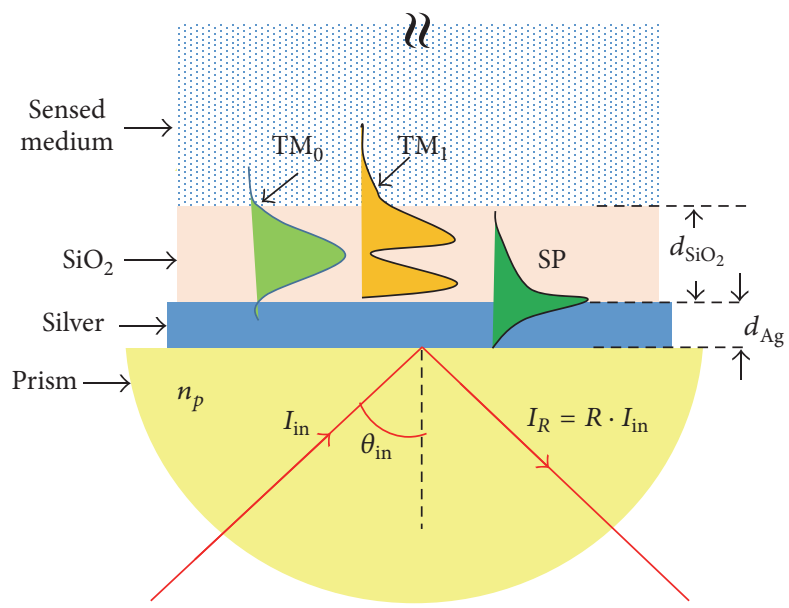

(a)

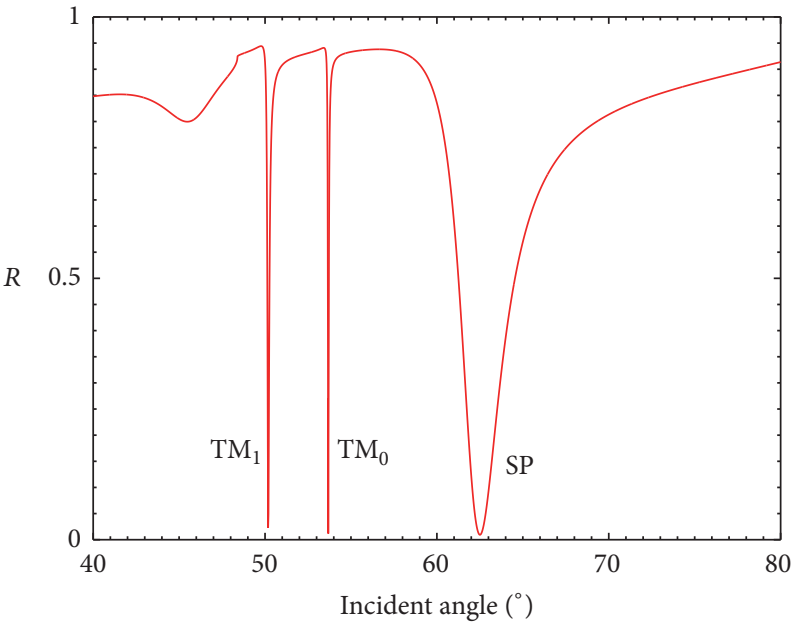

(b)

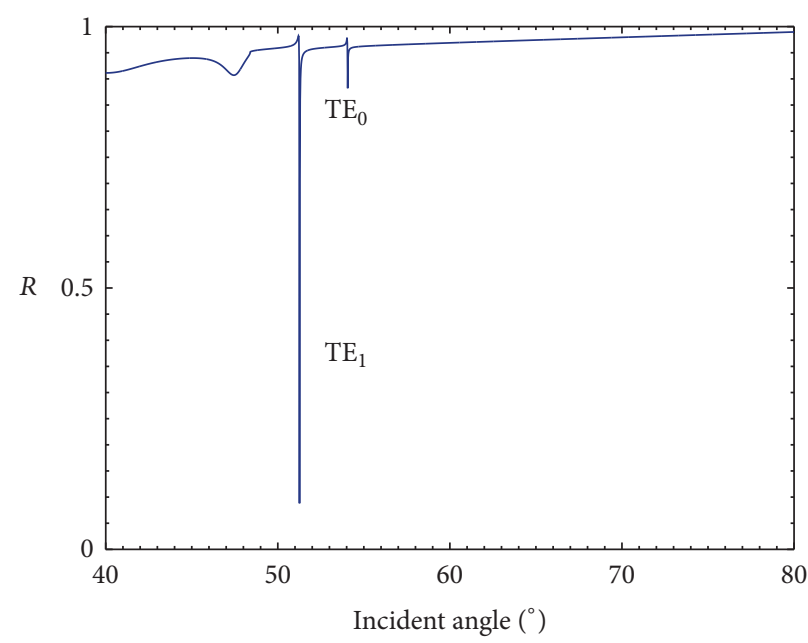

(c)

FIGURE 1: (a) Schematic illustration of the CPWR configuration that is used in the present study showing the structure composition and qualitative description of the different modes distribution inside the layers; (b) reflectivity versus incident angle for excitation wavelength $\lambda=632.8 \mathrm{~nm}$, showing the first two guided modes and the SP mode when the incident beam is TM polarized; (c) reflectivity versus incidence angle for TE polarization at wavelength $\lambda=632.8 \mathrm{~nm}$ and the layers thicknesses given in the text.

orders at $\lambda_{\mathrm{TM} 0}=1268 \mathrm{~nm}$ and $\lambda_{\mathrm{TM} 1}=608 \mathrm{~nm}$, respectively, as shown in Figure $4(\mathrm{a})$. The $\mathrm{TE}_{0}$ and $\mathrm{TE}_{1}$ modes, on the other hand, are less intense and appear at $747 \mathrm{~nm}$ and $2270 \mathrm{~nm}$, respectively (see Figure 4(c)). The guided modes are spectrally narrow and well defined and can be continuously tuned in the NIR region by controlling the $\mathrm{SiO}_{2}$ layer thickness. In the SPR case, on the other hand, we observe two dips that are generated due to the prism dispersion as was previously shown by Shalabney and Abdulhalim [25]. The dispersion of the prism in this spectral region leads to bending in the dispersion branch of the SPR mode as shown in Figure 4(c), and thereby two possible resonances are observed as indicated by the arrows. These two intersection points correspond to the short wavelength SPR (SWLSP) and long wavelength SPR (LWSPR) that appear as two distinguishable dips in the reflectivity spectrum as observed in Figure 4(a). Under small variations in the analyte RI or in the incidence angle, these two dips will superimpose leading to producing an extremely broad dip, which strongly limits the possibilities for sensing. This limitation occurs in most of the practical applications of SPR in the spectral domain due to the inherent dispersion of the prism coupler, normally used in these structures.

In Figure 4(d) we compare the FWHM and FOM of the $\mathrm{TM}_{0}$ guided mode in the CPWR configuration with that of the conventional SPR (without the $\mathrm{SiO}_{2}$ layer) when they are excited at the same wavelengths. We found that the width of the $\mathrm{TM}_{0}$ mode (full diamonds) is at least one order of magnitude smaller than that of the conventional SPR (full circles) when they are excited at the same wavelengths. Moreover, the width of the guided mode is almost constant on the entire spectral range whereas the width of the SPR 


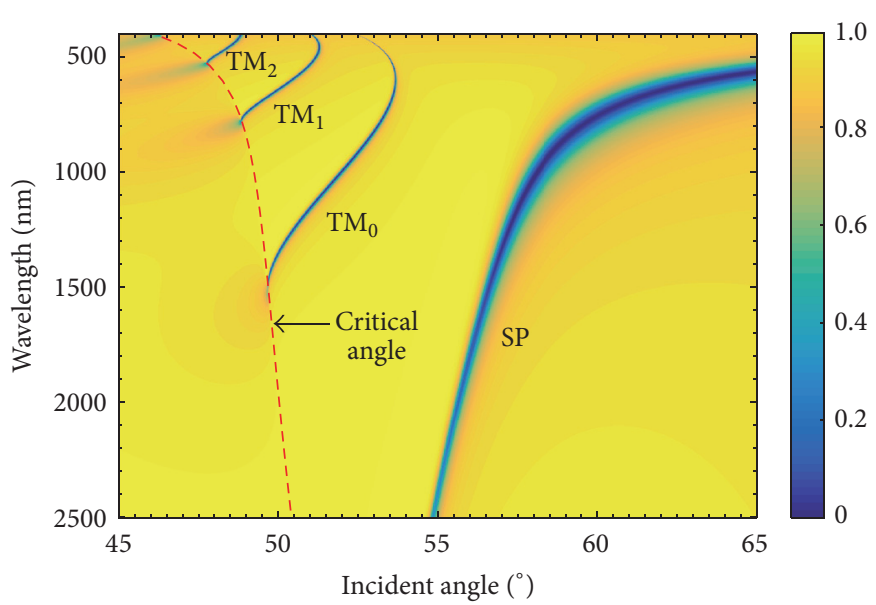

(a)

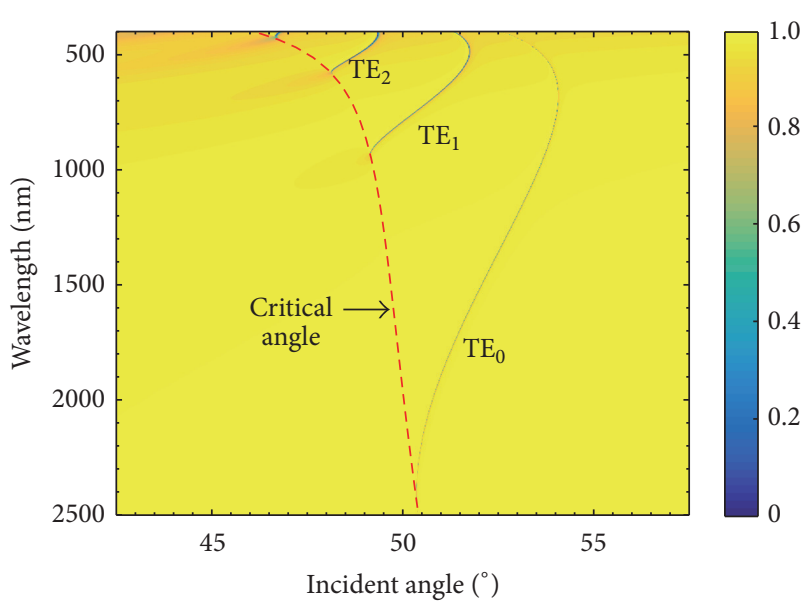

(b)

FIGURE 2: (a) Dispersion map of the TM polarized modes showing the branches of the different excited modes (see details in the text); (b) dispersion map of the TE polarized modes. The dashed red line in both panels shows the critical angle curve which originates from the prism dispersion in the interrogated spectral range.

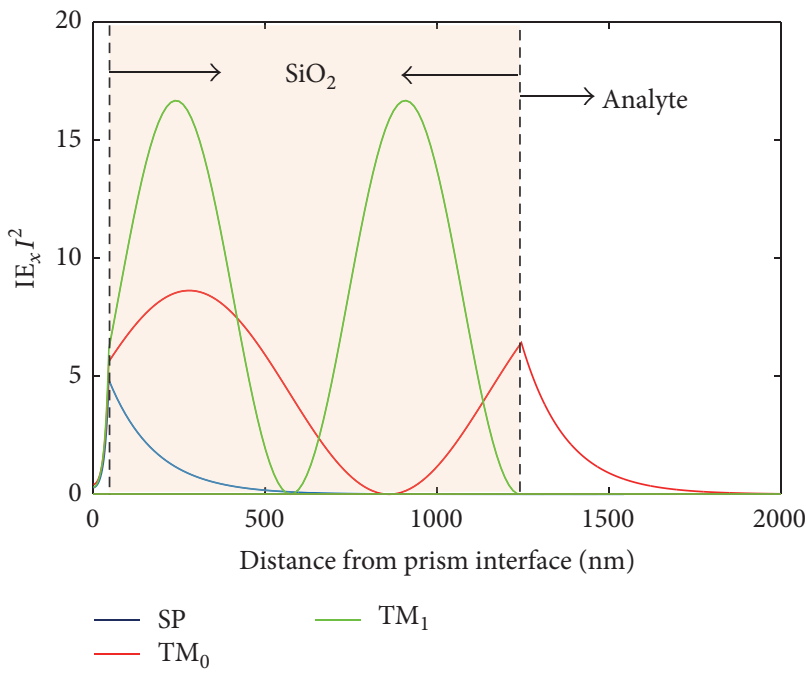

(a)

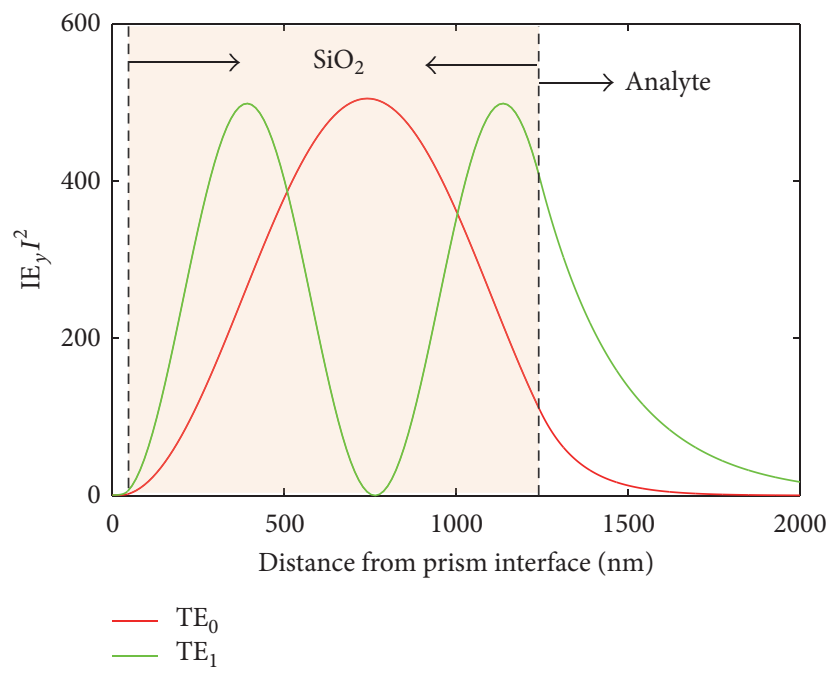

(b)

Figure 3: (a) The intensity of the $x$-component of the electric field versus the distance from the prism interface. (b) The intensity of the $y$-component of the electric field versus the distance from the prism interface. The fields are calculated for excitation wavelength $\lambda=785 \mathrm{~nm}$. All the intensities are normalized to the incident field.

strongly increases with the wavelength. The widths of the TE modes (Figure 4(c)) are even narrower than the TM and can reach two orders of magnitude smaller than that of the conventional SPR. Because of the narrow spectral widths, the guided modes exhibit an enhanced FOM (opened diamonds in Figure 4(d)) with respect to the SPR (opened circles), in particular in the NIR region. The FOM is normally defined as the ratio between the spectral sensitivity and the dip width $\left(\mathrm{FOM} \equiv S_{\lambda} / \mathrm{FWHM}\right)$. The steep increase in the FOM and the constant spectral width show that the sensitivity of the guided modes is significantly enhanced in the NIR region.
The extremely narrow line widths of both TM and TE guided modes and their high spectral sensitivity to changes in the analyte medium manifest their high potential for sensing, in particular in the NIR region. The anisotropy that characterizes cells and proteins, for instance, can be detected using the CPWR guided modes in the spectral interrogation approach. It is important to note that the penetration depths of the guided modes in the NIR region can reach few microns inside the analyte medium which is essential for cells and bacteria studies. These applications are of high relevance for biophysicists due to the deterministic correlation between 


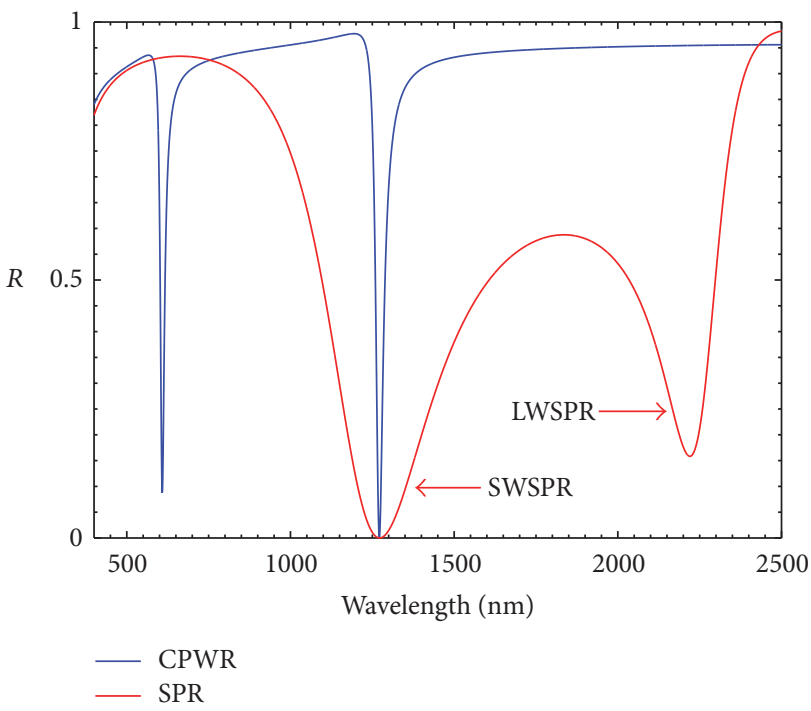

(a)

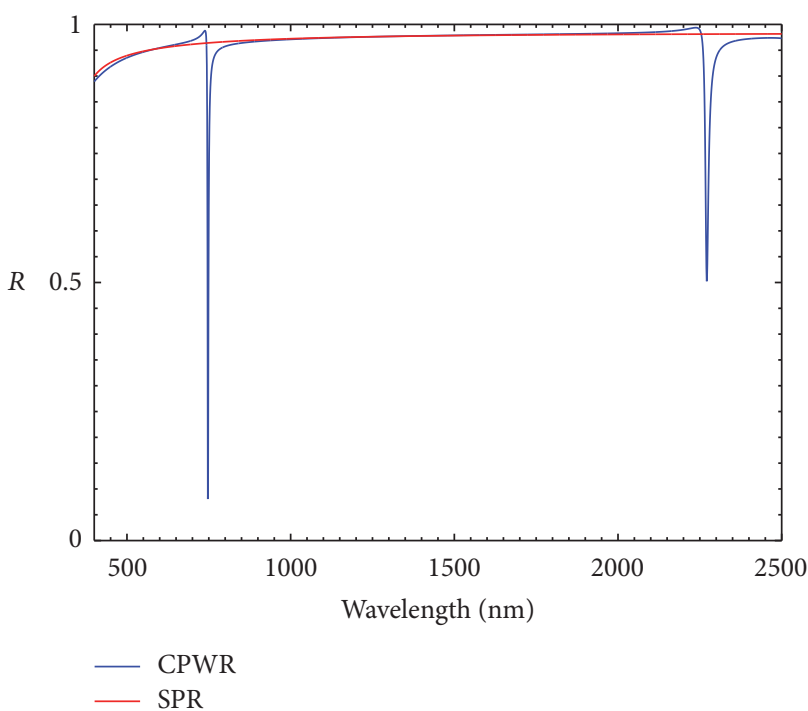

(c)

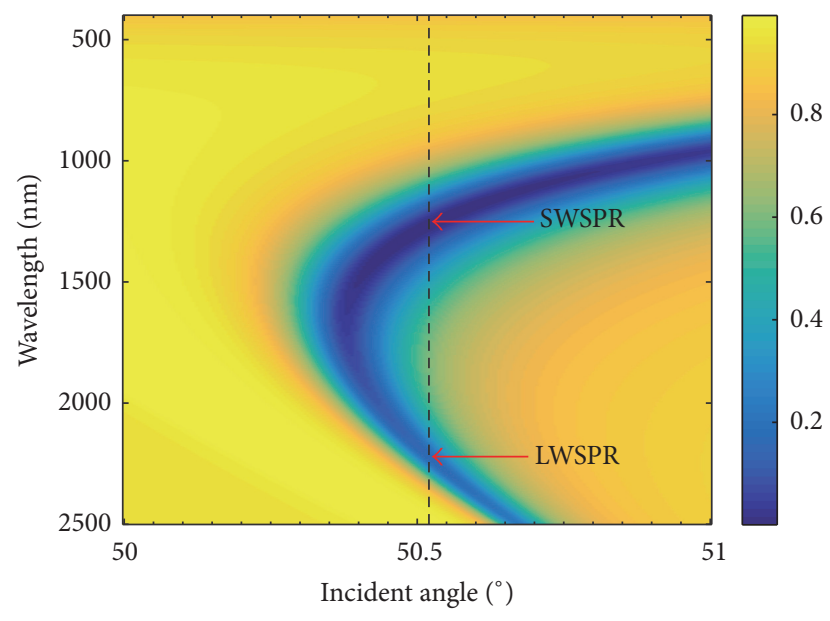

(b)

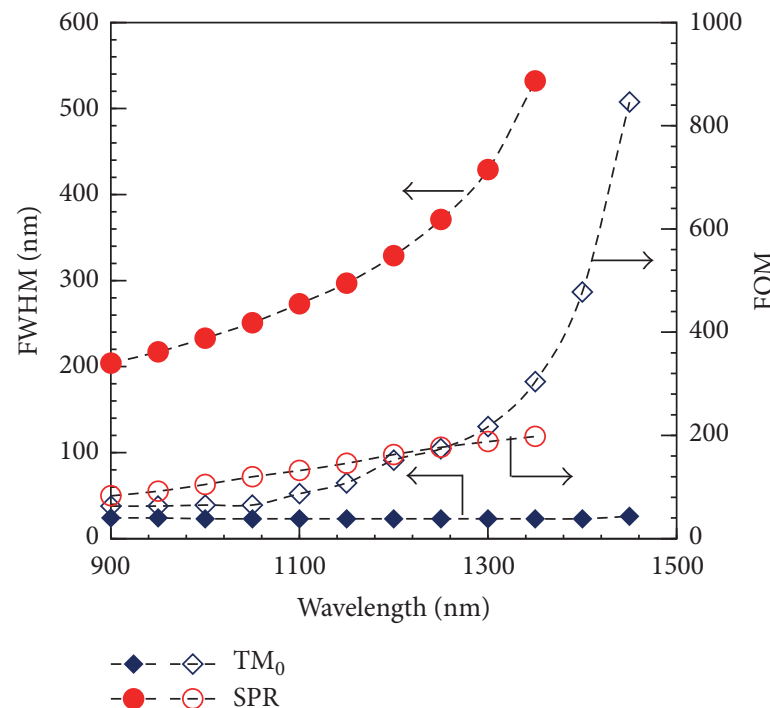

(d)

FIGURE 4: Spectral reflectivity of the guided modes in the CPWR configuration versus the reflectivity of conventional $\mathrm{SPR}_{\mathrm{R}}$ without the $\mathrm{SiO}_{2}$ dielectric layer for (a) TM polarization and (c) TE polarization. The incidence angle in (a) and (c) was tuned to 50.44 ${ }^{\circ}$; (b) dispersion branch of the conventional SPR mode in a reduced region, showing the bending effect due to the prism dispersion. The arrows show the appearance of the LWSPR and SWSPR modes at $50.44^{\circ}$ that correspond to the two dips shown in (a). (d) Full and opened diamonds show, respectively, the FWHM and FOM of the $\mathrm{TM}_{0}$ mode whereas the filled and opened circles show the FWHM and FOM of the conventional SPR.

the structural features of large biological entities and their functionality $[19,26,27]$.

\section{Conclusions}

We theoretically studied the nature and properties of the guided modes excited in the CPWR configuration. By calculating the full dispersion of the TM and TE polarization states, we characterized the angular and spectral behavior of these modes and their dependence on the structure. We found that these modes could be excited in the spectral interrogation method and easily tuned in the NIR region with high spectral sensitivity. Moreover, their extremely narrow spectral bandwidths and their polarization dependence make these resonances very promising for anisotropic biosensing where information pertinent to structural deformation is of high interest. Using the spectral interrogation method in SPR sensors is becoming more and more attractive due to the availability of miniature and cheap spectrometers and due to the importance in combining the SPR sensors with absorption spectroscopy for molecular recognition. Using 
the guided modes in the CPWR configuration enables the possibility of sensing in the NIR region without the limitation of resonances broadening which is inherent property in conventional SPR sensors.

\section{Competing Interests}

The authors declare that there is no conflict of interests regarding the publication of this paper.

\section{References}

[1] J. Homola, S. S. Yee, and G. Gauglitz, "Surface plasmon resonance sensors: review," Sensors and Actuators B: Chemical, vol. 54, no. 1-2, pp. 3-15, 1999.

[2] A. V. Kabashin and P. I. Nikitin, "Surface plasmon resonance interferometer for bio- and chemical-sensors," Optics Communications, vol. 150, no. 1-6, pp. 5-8, 1998.

[3] A. Shalabney and I. Abdulhalim, "Sensitivity-enhancement methods for surface plasmon sensors," Laser \& Photonics Reviews, vol. 5, no. 4, pp. 571-606, 2011.

[4] J. Homola, "On the sensitivity of surface plasmon resonance sensors with spectral interrogation," Sensors and Actuators B: Chemical, vol. 41, no. 1-3, pp. 207-211, 1997.

[5] G. Gupta and J. Kondoh, "Tuning and sensitivity enhancement of surface plasmon resonance sensor," Sensors and Actuators, B: Chemical, vol. 122, no. 2, pp. 381-388, 2007.

[6] J. S. Yuk, D.-G. Hong, J.-W. Jung et al., "Sensitivity enhancement of spectral surface plasmon resonance biosensors for the analysis of protein arrays," European Biophysics Journal, vol. 35, no. 6, pp. 469-476, 2006.

[7] X.-C. Yuan, B. H. Ong, Y. G. Tan, D. W. Zhang, R. Irawan, and S. C. Tjin, "Sensitivity-stability-optimized surface plasmon resonance sensing with double metal layers," Journal of Optics A: Pure and Applied Optics, vol. 8, no. 11, pp. 959-963, 2006.

[8] A. Lahav, M. Auslander, and I. Abdulhalim, "Sensitivity enhancement of guided-wave surface-plasmon resonance sensors," Optics Letters, vol. 33, no. 21, pp. 2539-2541, 2008.

[9] A. Lahav, A. Shalabaney, and I. Abdulhalim, "Surface plasmon sensor with enhanced sensitivity using top nano dielectric layer," Journal of Nanophotonics, vol. 3, no. 1, Article ID 031501, 2009.

[10] A. Shalabney and I. Abdulhalim, "Figure-of-merit enhancement of surface plasmon resonance sensors in the spectral interrogation," Optics Letters, vol. 37, no. 7, pp. 1175-1177, 2012.

[11] P. Bhatia and B. D. Gupta, "Surface-plasmon-resonance-based fiber-optic refractive index sensor: sensitivity enhancement," Applied Optics, vol. 50, no. 14, pp. 2032-2036, 2011.

[12] S. Y. Wu, H. P. Ho, W. C. Law, C. Lin, and S. K. Kong, "Highly sensitive differential phase-sensitive surface plasmon resonance biosensor based on the Mach-Zehnder configuration," Optics Letters, vol. 29, no. 20, pp. 2378-2380, 2004.

[13] A. Shalabney, A. Lakhtakia, I. Abdulhalim et al., "Surface plasmon resonance from metallic columnar thin films," Photonics and Nanostructures_Fundamentals and Applications, vol. 7, no. 4, pp. 176-185, 2009.

[14] A. Shalabney, C. Khare, B. Rauschenbach, and I. Abdulhalim, "Sensitivity of surface plasmon resonance sensors based on metallic columnar thin films in the spectral and angular interrogations," Sensors and Actuators B: Chemical, vol. 159, no. 1, pp. 201-212, 2011.
[15] Z. Salamon, H. A. Macleod, and G. Tollin, "Coupled plasmonwaveguide resonators: a new spectroscopic tool for probing proteolipid film structure and properties," Biophysical Journal, vol. 73, no. 5, pp. 2791-2797, 1997.

[16] Z. Salamon, G. Lindblom, L. Rilfors, K. Linde, and G. Tollin, "Interaction of phosphatidylserine synthase from E. coli with lipid bilayers: coupled plasmon-waveguide resonance spectroscopy studies," Biophysical Journal, vol. 78, no. 3, pp. 1400$1412,2000$.

[17] Z. Salamon, M. F. Brown, and G. Tollin, "Plasmon resonance spectroscopy: probing molecular interactions within membranes," Trends in Biochemical Sciences, vol. 24, no. 6, pp. 213219, 1999.

[18] Z. Salamon, G. Lindblom, and G. Tollin, "Plasmon-waveguide resonance and impedance spectroscopy studies of the interaction between penetratin and supported lipid bilayer membranes," Biophysical Journal, vol. 84, no. 3, pp. 1796-1807, 2003.

[19] E. Harté, N. Maalouli, A. Shalabney et al., "Probing the kinetics of lipid membrane formation and the interaction of a nontoxic and a toxic amyloid with plasmon waveguide resonance," Chemical Communications, vol. 50, no. 32, pp. 4168-4171, 2014.

[20] N. Skivesen, R. Horvath, and H. C. Pedersen, "Optimization of metal-clad waveguide sensors," Sensors and Actuators B: Chemical, vol. 106, no. 2, pp. 668-676, 2005.

[21] V. Yashunsky, V. Lirtsman, M. Golosovsky, D. Davidov, and B. Aroeti, "Real-time monitoring of epithelial cell-cell and cell-substrate interactions by infrared surface plasmon spectroscopy," Biophysical Journal, vol. 99, no. 12, pp. 4028-4036, 2010.

[22] I. D. Alves, D. Delaroche, B. Mouillac et al., "The two NK1 binding sites correspond to distinct, independent, and noninterconvertible receptor conformational states as confirmed by plasmon-waveguide resonance spectroscopy," Biochemistry, vol. 45, no. 16, pp. 5309-5318, 2006.

[23] I. D. Alves, I. Correia, C. Y. Jiao et al., "The interaction of cell-penetrating peptides with lipid model systems and subsequent lipid reorganization: thermodynamic and structural characterization," Journal of Peptide Science, vol. 15, no. 3, pp. 200-209, 2009.

[24] A. Shalabney and I. Abdulhalim, "Electromagnetic fields distribution in multilayer thin film structures and the origin of sensitivity enhancement in surface plasmon resonance sensors," Sensors and Actuators A: Physical, vol. 159, no. 1, pp. 24-32, 2010.

[25] A. Shalabney and I. Abdulhalim, "Prism dispersion effects in near-guided-wave surface plasmon resonance sensors," Annalen der Physik, vol. 524, no. 11, pp. 680-686, 2012.

[26] G. Haran, "How, when and why proteins collapse: the relation to folding," Current Opinion in Structural Biology, vol. 22, no. 1, pp. 14-20, 2012.

[27] R. Sarroukh, E. Goormaghtigh, J.-M. Ruysschaert, and V. Raussens, "ATR-FTIR: a 'rejuvenated' tool to investigate amyloid proteins," Biochimica et Biophysica Acta (BBA)_Biomembranes, vol. 1828, no. 10, pp. 2328-2338, 2013. 


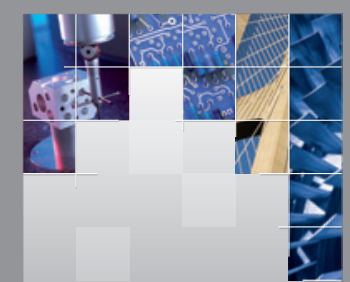

\section{Enfincering}
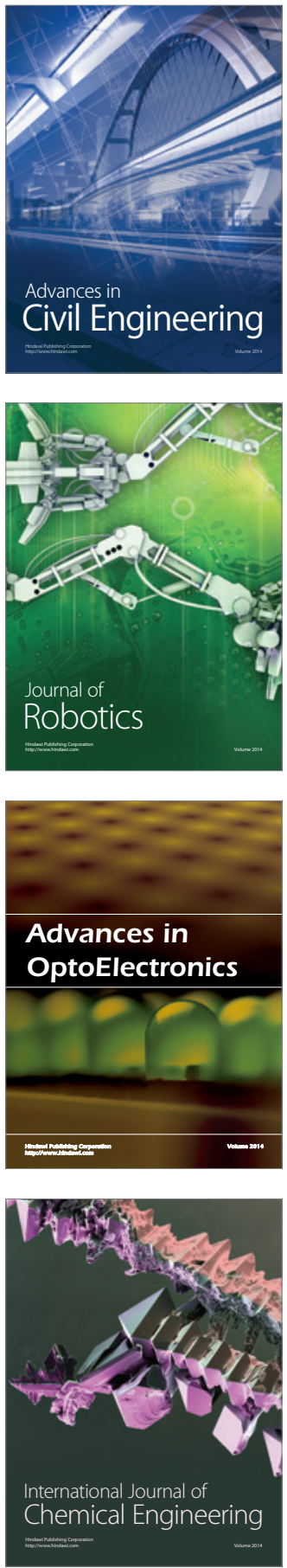

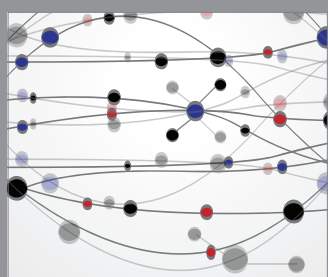

The Scientific World Journal

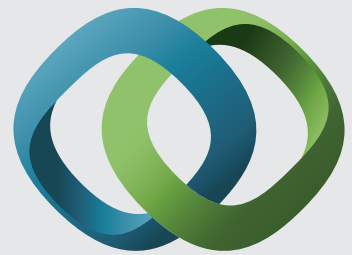

\section{Hindawi}

Submit your manuscripts at

http://www.hindawi.com
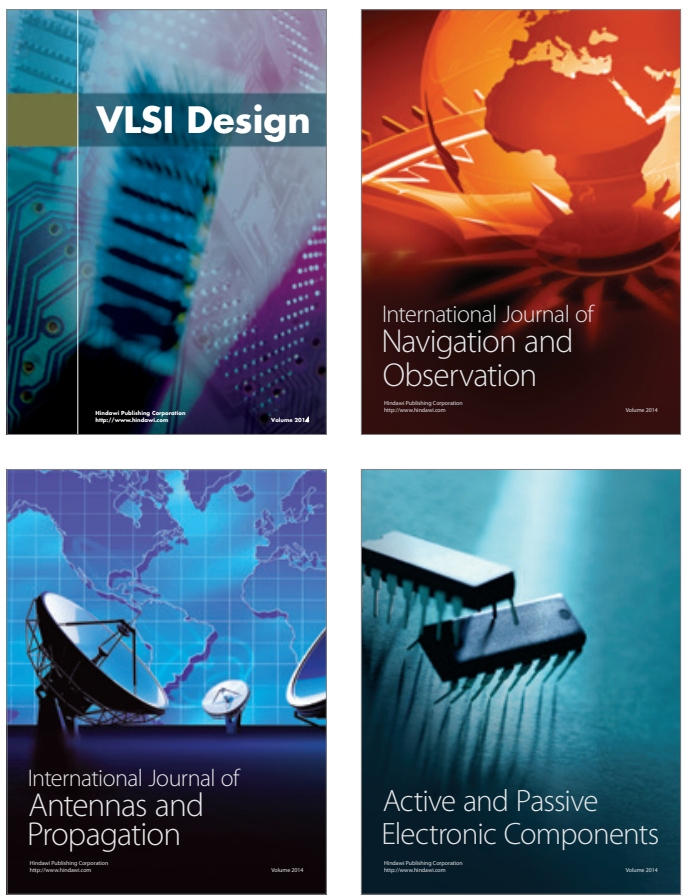
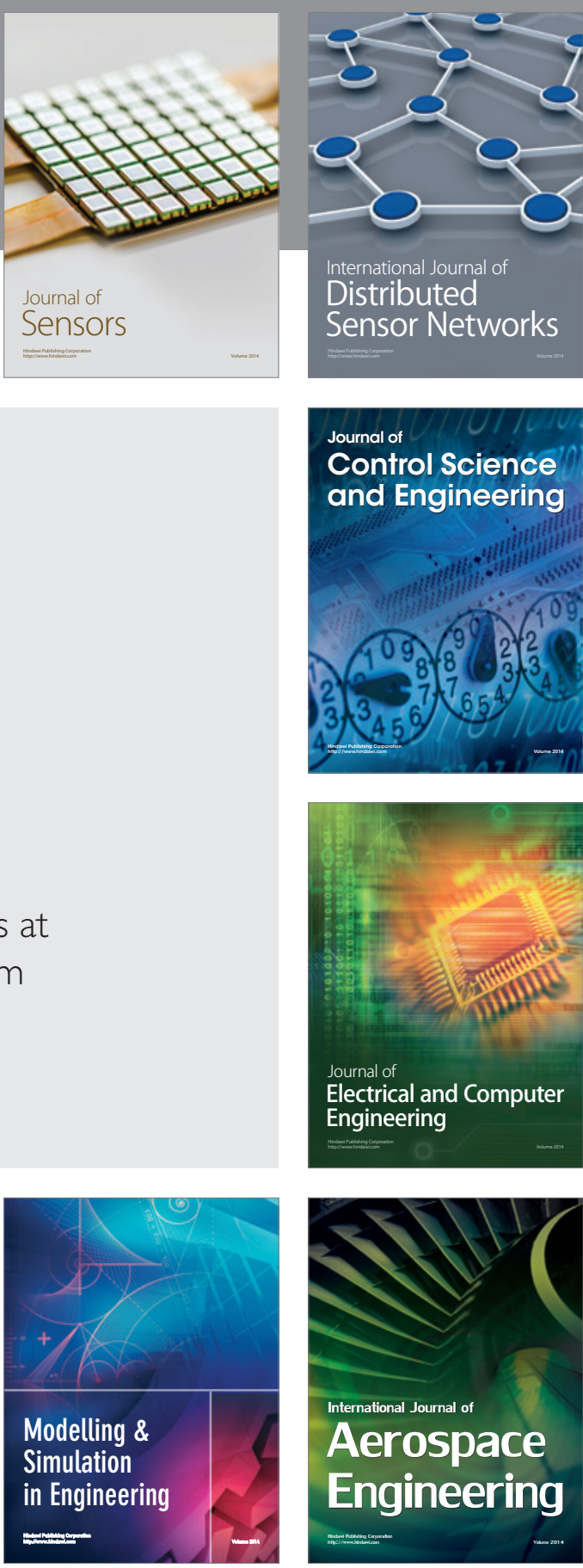

International Journal of

Distributed

Sensor Networks

Journal of

Control Science

and Engineering
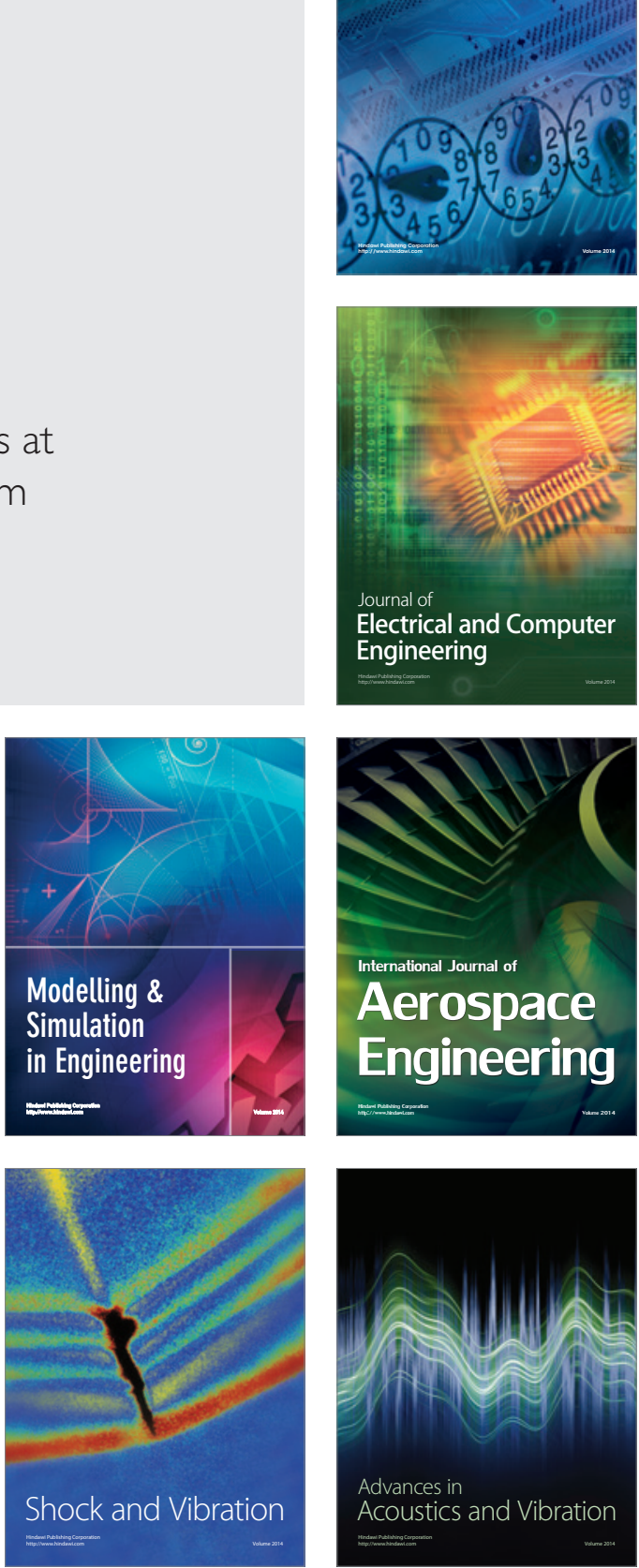